\title{
Differential Evolution for the Control Gain's Optimal Tuning of a Four-bar Mechanism
}

\author{
María Bárbara Calva-Yáñez, Paola Andrea Niño-Suárez, Miguel Gabriel Villarreal-Cervantes, \\ Gabriel Sepúlveda-Cervantes, and Edgar Alfredo Portilla-Flores
}

\begin{abstract}
In this paper the variation of the velocity error of a four-bar mechanism with spring and damping forces is reduced by solving a dynamic optimization problem using a differential evolution algorithm with a constraint handling mechanism. The optimal design of the velocity control for the mechanism is formulated as a dynamic optimization problem. Moreover, in order to compare the results of the differential evolution algorithm, a simulation experiment of the proposed control strategy was carried out. The simulation results and discussion are presented in order to evaluate the performance of both approaches in the control of the mechanism.
\end{abstract}

Index Terms-Velocity control, differential evolution algorithm, four-bar mechanism, dynamic optimization.

\section{INTRODUCTION}

$\mathbf{T}$ HE four-bar mechanism (FBM) is extensively used in several engineering applications [1], [2], [3]. This is due to the topological simplicity, functional versatility and because this mechanism can generate a cyclic trajectory (path generation). Hence, the four-bar mechanism has been widely studied in the last decades. The path generation of the four-bar mechanism is achieved by using analytical, numerical and graphical methods [4]-[6]. Nevertheless, the statement of optimization problems to increase the number of precision points and the tracking precision are been used in the path generation of the FBM [7], [8].

In the analysis and design of the FBM the main assumption considers that the angular velocity of the actuator is constant. Nevertheless, it is not always fulfilled, if an electric motor drives the crank. For example, when the crank rotates, the center of mass of the FBM may move. The change of the inertia of the FBM yield an external load to the motor such that the angular velocity of the crank is not constant. Hence, it is important to select the appropriate control system that guarantee an uniform and efficient regulation of the angular velocity.

Manuscript received on February 25, 2013; accepted for publication on May 23, 2013.

M. B. Calva-Yáñez, M. G. Villarreal-Cervantes, G. Sepúlveda-Cervantes, and E. A. Portilla-Flores are with the Instituto Politécnico Nacional, CIDETEC, Mechatronic Section, Postgraduate Department, Juan de Dios Bátiz s/n, C.P. 07700 D.F., Mexico (e-mail: b_calva@hotmail.com; $\{$ mvillarrealc, gsepulveda, aportilla\}@ipn.mx).

P. A. Niño-Suárez is with the Instituto Politécnico Nacional, ESIME-AZC, SEPI Section, Las Granjas 682, C.P. 02250 D.F., Mexico (e-mail: pninos@ipn.mx).
There exist several advanced control strategy that may guarantee robustness in the angular velocity, such as robust control [9], adaptive control [10], etc. Nevertheless, from an industrial point of view, PID controllers can provide a good performance if the gains are correctly tuned in.

In this paper, the modified PID controller presented in [11] is used to regulate the angular velocity of a four-bar mechanism with spring and damping forces (FBM-SDF). The optimal PID control gains is found by considering a dynamic optimization problem and by using a constraint handling mechanism in the differential evolution algorithm to solve it. The effectiveness of the algorithm is shown in simulation results.

The paper is organized as follows: Section II presents the coupled dynamics of the four-bar mechanism with DC motor. Section III presents the control strategy for the system. In Section IV, the dynamic optimization problem is stated for finding the optimal control gains. The constraint handling differential evolution algorithm is show in Section V. The simulation results and discussion are given in Section VI and finally, the conclusions are drawn in Section VII.

\section{DyNAMIC MODEL}

The four-bar mechanism with spring and damping forces (FBM-SDF) has one degree of freedom (dof) in the crank (link $L_{2}$ ). This dof is actuated by a DC motor. The schematic representation of the mechanism is shown in Fig. 1. The mass, the inertia, the length, the mass center length and the mass center angle of the $i$-th link are represented by $m_{i}, J_{i}, L_{i}, r_{i}, \phi_{i}$, respectively. The angle of the $i$-th link with respect to the base frame (X-Y) is named as $\theta_{i}$. The stiffness constant of the spring and the damping coefficient of the damper are represented by $k$ and $C$.

The kinematic analysis [5] of the FBM-SDF is required to obtain the angular velocity $\dot{\theta}_{i} \forall i=2,3,4$ and the linear velocity $v_{i x}, v_{i y}$ of the mass center of the $i$-th link with respect to the inertial frame. The angular and the linear velocity is described in (1)-(3).

$$
\begin{aligned}
\dot{\theta}_{i} & =\gamma_{i} \dot{\theta}_{2} \\
v_{i x} & =\alpha_{i} \dot{\theta}_{2} \\
v_{i y} & =\beta_{i} \dot{\theta}_{2}
\end{aligned}
$$




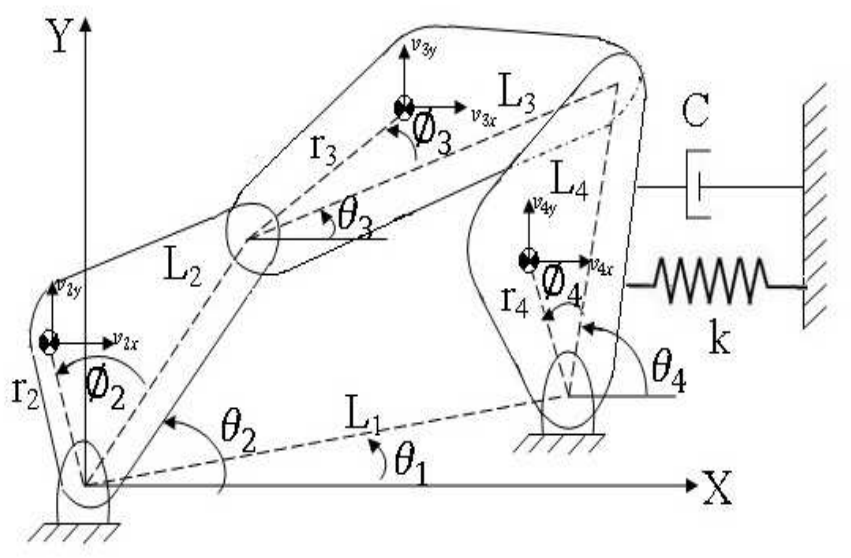

Fig. 1. Four-bar mechanism with spring and damping forces

where:

$$
\begin{aligned}
\alpha_{2} & =-r_{2} \sin \left(\theta_{2}+\phi_{2}\right) \\
\alpha_{3} & =-L_{2} \sin \theta_{2}-r_{3} \gamma_{3} \sin \left(\theta_{3}+\phi_{3}\right) \\
\alpha_{4} & =-r_{4} \gamma_{4} \sin \left(\theta_{4}+\phi_{4}\right) \\
\beta_{2} & =r_{2} \cos \left(\theta_{2}+\phi_{2}\right) \\
\beta_{3} & =L_{2} \cos \theta_{2}-r_{3} \gamma_{3} \cos \left(\theta_{3}+\phi_{3}\right) \\
\beta_{4} & =-r_{4} \gamma_{4} \cos \left(\theta_{4}+\phi_{4}\right) \\
\gamma_{2} & =1 \\
\gamma_{3} & =\frac{L_{2} \sin \left(\theta_{4}-\theta_{2}\right)}{L_{3} \sin \left(\theta_{3}-\theta_{4}\right)} \\
\gamma_{4} & =\frac{L_{2} \sin \left(\theta_{3}-\theta_{2}\right)}{L_{3} \sin \left(\theta_{3}-\theta_{4}\right)}
\end{aligned}
$$

Defining the Lagrangian function $\tilde{L}(13)$, where $K$ and $P$ is the kinetic and potential energy, respectively.

$$
\tilde{L}=K-P
$$

where:

$$
\begin{aligned}
& K=\sum_{i=2}^{4}\left(\frac{1}{2} m_{i}\left(v_{i x}^{2}+v_{i y}^{2}\right)+\frac{1}{2} J_{i} \dot{\theta}_{i}^{2}\right)=\frac{1}{2} A\left(\theta_{2}\right) \dot{\theta}_{2}^{2} \\
& P=\frac{1}{2} k\left(\theta_{4}-\theta_{4,0}\right)^{2} \\
& A\left(\theta_{2}\right)=\sum_{i=2}^{4}\left(m_{i}\left(\alpha_{i}^{2}+\beta_{i}^{2}\right)+\gamma_{i}^{2} J_{i}\right)
\end{aligned}
$$

Using $\theta_{2}$ as the generalized coordinate and following the methodology described in [11], the Euler-Lagrange formulation [12] which described the dynamic model of the FBM-SDF is given by (17), where $D$ is the Rayleigh's dissipation function and $\theta_{4,0}$ is the angular position of the link 4 when the spring is in equilibrium.

$$
T=\frac{d}{d t}\left(\frac{\partial \tilde{L}}{\partial \dot{\theta}_{2}}\right)-\frac{\partial \tilde{L}}{\partial \theta_{2}}+\frac{\partial D}{\partial \dot{\theta}_{2}}
$$

where:

$$
D=\frac{1}{2} C \dot{\theta}_{4}^{2}
$$

The total and partial derivatives of (17) is given by (19).

$$
T=A\left(\theta_{2}\right) \ddot{\theta}_{2}+\frac{1}{2} \frac{d A\left(\theta_{2}\right)}{d \theta_{2}} \dot{\theta}_{2}^{2}+k \gamma_{4}\left(\theta_{4}-\theta_{4,0}\right)+C \gamma_{4}^{2} \dot{\theta}_{2}
$$

where:

$$
A\left(\theta_{2}\right)=C_{0}+C_{1} \gamma_{3}^{2}+C_{2} \gamma_{4}^{2}+C_{3} \gamma_{3} \cos \left(\theta_{2}-\theta_{3}-\phi_{3}\right)
$$

$$
\begin{aligned}
& \frac{d A\left(\theta_{2}\right)}{d \theta_{2}}=2 C_{1} \gamma_{3} \frac{d \gamma_{3}}{d \theta_{2}}+2 C_{2} \gamma_{4} \frac{d \gamma_{4}}{d \theta_{2}} \\
& +C_{3} \frac{d \gamma_{3}}{d \theta_{2}} \cos \left(\theta_{2}-\theta_{3}-\phi_{3}\right) \\
& -C_{3} \gamma_{3}\left(1-\gamma_{3}\right) \sin \left(\theta_{2}-\theta_{3}-\phi_{3}\right) \\
& C_{0}=J_{2}+m_{2} r_{2}^{2}+m_{3} L_{2}^{2} \\
& C_{1}=J_{3}+m_{3} r_{3}^{2} \\
& C_{2}=J_{4}+m_{4} r_{4}^{2} \\
& C_{4}=2 m_{3} L_{2} r_{3} \\
& \frac{d \gamma_{3}}{d \theta_{2}}=\frac{L_{2}\left(D_{1}+D_{2}\right)}{L_{3} \sin ^{2}\left(\theta_{3}-\theta_{4}\right)} \\
& \frac{d \gamma_{4}}{d \theta_{2}}=\frac{L_{2}\left(D_{3}+D_{4}\right)}{L_{4} \sin ^{2}\left(\theta_{3}-\theta_{4}\right)} \\
& D_{1}=\left(\gamma_{4}-1\right) \sin \left(\theta_{3}-\theta_{4}\right) \cos \left(\theta_{4}-\theta_{2}\right) \\
& D_{2}=\left(\gamma_{4}-\gamma_{3}\right) \sin \left(\theta_{4}-\theta_{2}\right) \cos \left(\theta_{3}-\theta_{4}\right) \\
& D_{3}=\left(\gamma_{3}-1\right) \sin \left(\theta_{3}-\theta_{4}\right) \cos \left(\theta_{3}-\theta_{2}\right) \\
& D_{4}=\left(\gamma_{4}-\gamma_{3}\right) \sin \left(\theta_{3}-\theta_{2}\right) \cos \left(\theta_{3}-\theta_{4}\right)
\end{aligned}
$$

In order to model the full dynamics of the FBM-SDF, the dynamic of the actuator [13] must be included. A schematic diagram of the DC motor is represented in Fig. 2, where $L$ and $R$ represent the inductance and the armature resistance, $i(t)$ and $V_{i n}(t)$ are the current and voltage input, respectively. $J$ and $B$ is the inertia moment and the friction coefficient of the output shaft. $T_{L}, T_{m}$ and $V_{b}$ is the load torque, the magnetic motor torque and the Back electromotive force of the motor, respectively. The motor constant is represented by $K_{f}$ and the constant of the back electromotive force is represented by $K_{b}$.

The dynamic model of the DC motor [14] consists on modeling the electrical and mechanical parts. Using Kirchhoff's second law, the closed loop circuit of Fig. 2 can be written as (32).

$$
L \frac{d i(t)}{d t}+R i(t)=V_{i n}(t)-K_{b} \dot{\theta}_{1}
$$

By using the Newton's second law in the mechanical part of the DC motor, the equation (33) is obtanied, where $T_{a}$ and 


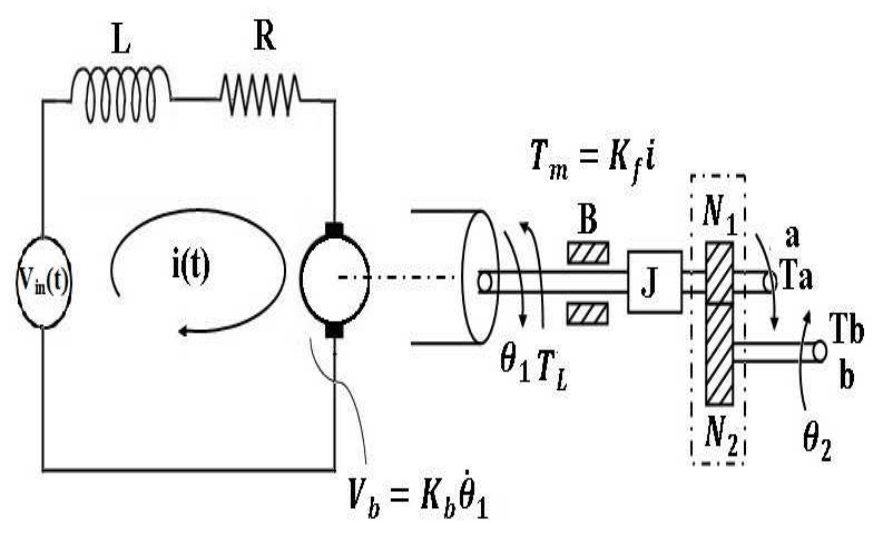

Fig. 2. Schematic diagram of a DC motor

$T_{b}$ is the output torque of the shaft $a$ and $b$, respectively (see Fig. 2).

$$
T_{m}-B \dot{\theta}_{1}-T_{a}-T_{L}=J \ddot{\theta}_{1}
$$

The mechanical transmission among the two gears in the shafts is expressed in (34), where $r_{i}$ and $N_{i} \forall i=1,2$ is the radius and the number of teeth of the gears.

$$
\frac{T_{b}}{T_{a}}=\frac{\dot{\theta}_{1}}{\dot{\theta}_{2}}=\frac{r_{2}}{r_{1}}=\frac{N_{2}}{N_{1}}=n
$$

Substituting $T_{a}$ from (33) to (34), the torque applied to the mechanical system is written as (35).

$$
T_{b}=n\left(T_{m}-T_{L}-B \dot{\theta}_{1}-J \ddot{\theta}_{1}\right)
$$

Using the relation $\dot{\theta_{1}}=n \dot{\theta_{2}}$ in (34), $T_{m}=K_{f} i$ and $T_{L}=0$, the dynamic equation of the DC motor is given by (36)-(37).

$$
\begin{aligned}
& T_{b}=n K_{f} i(t)-n^{2} B \dot{\theta}_{2}-n^{2} J \ddot{\theta}_{2} \\
& L \frac{d i(t)}{d t}+R i(t)=V_{i n}(t)-n k_{b} \dot{\theta}_{2}
\end{aligned}
$$

Hence, the coupled dynamics of the DC motor with the FBM-SDF is given by combining (36), (37) and (19). Let the state variable vector $x=\left[x_{1}, x_{2}, x_{3}\right]^{T}=\left[\theta_{2}, \dot{\theta}_{2}, i\right]^{T}$ and the input vector $u=V_{i n}$, the coupled dynamics in a state space representation of the DC motor with the FBM-SDF is given by (38).

$$
\begin{aligned}
\dot{x} & =f(\vec{x}, u(t), t) \\
& =\left[\begin{array}{c}
x_{2} \\
A_{0}\left[A_{1} x_{2}^{2}+A_{2} x_{2}+n K_{f} x_{3}+A_{3}\right] \\
\frac{1}{L}\left(u(t)-n K_{b} x_{2}-R x_{3}\right)
\end{array}\right]
\end{aligned}
$$

where:

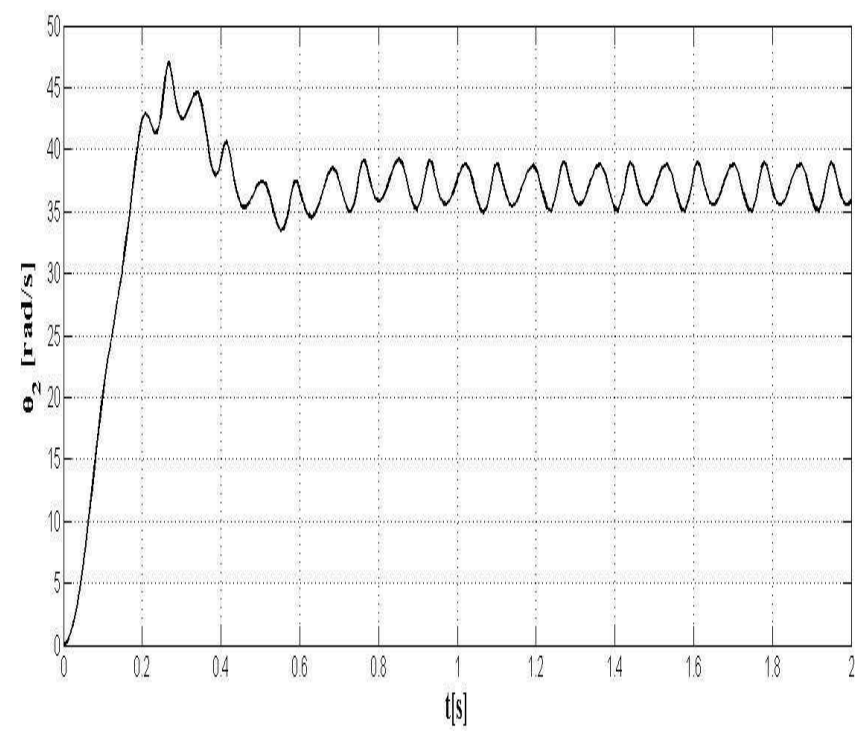

Fig. 3. Input angular velocity $\dot{\theta}_{2}$ of the FBM-SDF without a control strategy

$$
\begin{aligned}
A_{0} & =\frac{1}{A\left(x_{1}\right)+n^{2} J 1} \\
A_{1} & =-\frac{1}{2} \frac{d A\left(x_{1}\right)}{d x_{1}} \\
A_{2} & =-\left(C \gamma_{2}^{4}+n^{2} B\right) \\
A_{3} & =-k \gamma_{4}\left(\theta_{4}-\theta_{4,0}\right)
\end{aligned}
$$

\section{Control Strategy}

In the synthesis of mechanism, the main assumption is to consider the input velocity as a constant. Nevertheless, this can no be ensured without a closed loop control system. In Fig. 3 shows the behavior of the input angular velocity $\dot{\theta}_{2}$ when a constant voltage of $30 \mathrm{Volts}$ is applied. It is observed that the input angular velocity is not constant. This is true because the four-bar mechanism presents dead-centre positions and it adds uncertain loads in the crank.

Based on the work of Tao and Sadler [11], the proposed control strategy is used in this paper. This controller is stated as in (43), where $K_{p}, K_{I}$ and $K_{D}$ is the proportional (P), integral (I) and derivative (D) gains, respectively. The velocity error and its derivative are represented by $e(t)=\dot{\theta}_{2}^{d}-\dot{\theta}_{2}$ and $\dot{e}(t)=-\ddot{\theta}_{2}$, where $\dot{\theta}_{2}^{d}$ is the constant desired velocity.

$$
u(t)=K_{p} e(t) \int_{0}^{t} \dot{\theta}_{2}^{d} d t+K_{I} \int_{0}^{t} e(t) d t+K_{D} \dot{e}(t)
$$

\section{Dynamic Optimization Problem to Find the OPTIMUM CONTROLLER GAINS FOR CONSTANT INPUT VELOCITY OF THE FBM-SDF}

The dynamic optimization problem [15] consist on finding the optimum design variables $\vec{p} \in R^{3}$ such that minimize the 
objective function (44) subject to the closed-loop system of the FNM-SDF (45) with the initial state vector $x_{0}$, inequalities constraints (48) and bounds in the design variable (49).

$$
\min _{\vec{p} \in R^{3}} F(\vec{p})
$$

subject to:

$$
\begin{aligned}
& \dot{x}=f(\vec{x}, u(\vec{p}, t), t) \\
& u(t)=K_{p} e(t) \int_{0}^{t} \dot{\theta}_{2}^{d} d t+K_{I} \int_{0}^{t} e(t) d t+K_{D} \dot{e}(t) \\
& \vec{x}(0)=x_{0} \\
& g(\vec{x}) \leq 0 \\
& p_{i, \min } \leq \vec{p} \leq p_{i, \max }
\end{aligned}
$$

In the next subsections, variables, functions and all parts that involve the dynamic optimization problem (DOP) are described.

\section{A. Design Variable Vector}

The design variable vector $\vec{p}=\left[K_{p}, K_{D}, K_{I}\right]^{T} \in R^{3}$ includes the gains of the modified PID controller.

\section{B. Objective Function}

The variation of the input velocity of the crank is chosen as the objective function in the optimization problem. This is an important issue due to a bad selection of the PID gains, the input velocity of the crank could be considerably affected. The objective function is written as in (50), where $\operatorname{Max}()$ and $\operatorname{Min}()$ is the maximum and minimum value of the input velocity presented in the time interval $\left[0, t_{f}\right]$.

$$
F(\vec{p})=\left|\operatorname{Max}\left(x_{2}(t)\right)-\operatorname{Min}\left(x_{2}(t)\right)\right| ; t \in\left[0, t_{f}\right]
$$

\section{Constraints}

The first constraint (45) is the solution of the differential equation of the dynamic model of the FBM-SDF choosing $x_{0}$ as the initial condition. This constraints provide the dynamic behavior of the system in the optimization problem.

The inequality constraints consist on establishing that the rise time $t_{r}$ of the angular velocity of the crank $\dot{\theta}_{2}(t)$ is less than $0.1 s$ and the overshoot does not exceed of $1.7 \%$ of the desired angular velocity $\dot{\theta}_{2}^{d}$. These constraints is stated as in (51) and (52), respectively.

$$
\begin{aligned}
& g_{1}: t_{r} \leq 0.1 s \\
& g_{2}: \dot{\theta}_{2}\left(t_{r}\right) \leq \dot{\theta}_{2}^{d}+0.017 \dot{\theta}_{2}^{d}
\end{aligned}
$$

The bounds in the design variable vector are defined by $\vec{p}_{i, \min }$ and $\vec{p}_{i, \max } \forall i=1,2,3$.

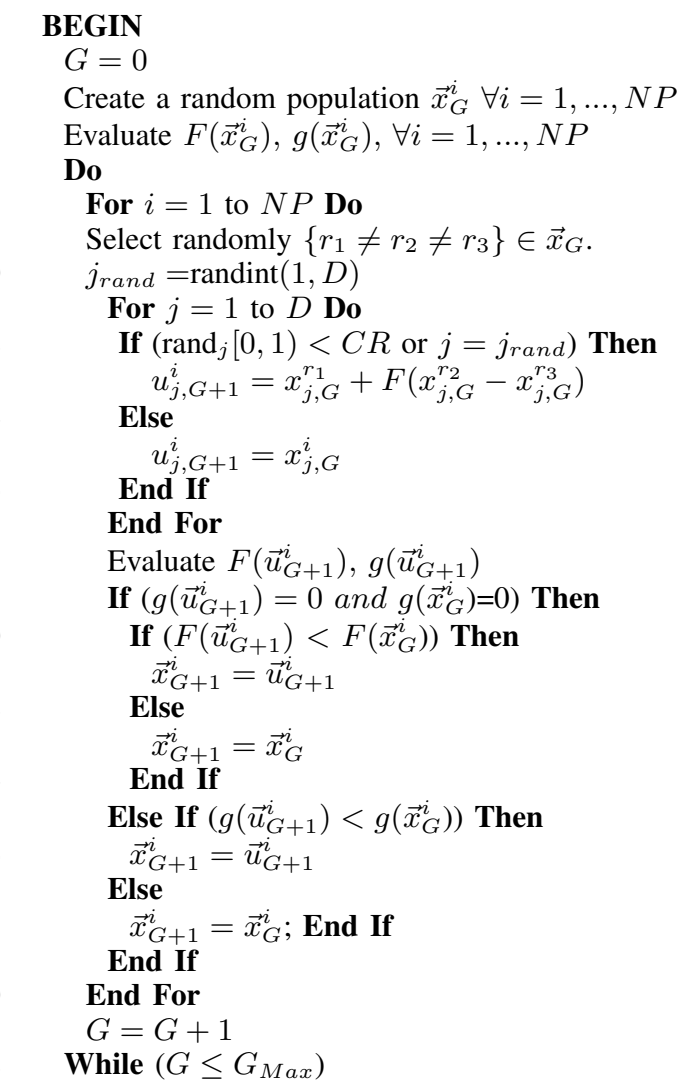

Fig. 4. CHDE algorithm

\section{Differential Evolution Algorithm}

In the last decades, the use of heuristic techniques have been used in engineering problems [16], [17]-[19]. This is due to the increment of the technological advances and because problems are non-convex, discontinuous and/or present discrete variables that make it difficult (or imposible) to solve them by traditional optimization techniques such as nonlinear programming techniques.

In this work, the differential evolution (DE) algorithm [20] with a constraint-handling mechanism [17] is used to solve the dynamic optimization problem. The constraint-handling differential evolution (CHDE) algorithm is shown in Fig. 4. The constraint handling mechanism is included in the selection operation between the trial vector $\vec{u}_{G+1}^{i}$ and the target vector $\vec{x}_{G}^{i}$ in order to remain one of them in the population for the next generation. This mechanism consists on passing the best individual between them for the next generation (elitism). The best individual is the individual without constraint violation and with less or equal objective function value or when both individuals are unfeasible, the best individual is the one with less constraint violation (see line 19 and 25 of Fig. 4).

For more details of the algorithm consult [20] and [17].

\section{Simulation Results}

The simulation results consist on using the CHDE algorithm in the dynamic optimization stated above. Four parameters 
in the CHDE algorithm must be chosen. In this case, the population size NP consists of 100 individuals. The scaling factor $F$ and the crossover constant $C R$ are randomly generated in the interval $F \in[0.3,0.9]$ at each generation, and in the interval $C R \in[0.8,1)$ at each optimization process. The stop criterion is when the number of generations is fulfilled $G_{\text {Max }}=200$.

The CHDE algorithm is programmed in Matlab Release 7.9 on a Windows platform. Computational experiments were performed on a PC with a $1.83 \mathrm{GHz}$ Core 2 Duo processor and $2 \mathrm{~GB}$ of RAM. Ten independent runs of the CHDE algorithm are performed.

On the other hand, in order to solve the dynamic optimization problem (44)-(49), the closed-loop system (45) must be solved numerically. Hence, the Runge-Kutta method (RKM) is used to solved it, with initial condition chosen as $x_{0}=[0,0,0]^{T}$, with a desired velocity selected as $\dot{\theta}_{2}^{d}=30$ $\mathrm{rad} / \mathrm{s}$ and with the kinematic and dynamic parameters of the coupled dynamics proposed as in Table I.

The bound of the design variable vector is defined as $\vec{p}_{i, \min }=0.1, \vec{p}_{i, \max }=50 \forall i=1,2,3$.

All runs of the algorithm converge to the optimum design variable vector $\vec{p}^{*}=[50,16.1881,1.4394]^{T}$ with a performance function value of $F\left(\vec{p}^{*}\right)=0.2389$. This means that local solutions are not found by the algorithm and the found solution can be considered as the global one. The mean of the time spends to converge the algorithm is ten minutes.

However, in order to compare the behavior of the system performance with the optimum design variable vector $\vec{p}^{*}$, the behavior of the system performance with PID gains obtained by a trial and error procedure is carried out. Such tuning is called experimental tuning in this paper. The experimental tuning considers the bounds $\vec{p}_{i, \text { min }}$ and $\vec{p}_{i, \text { max }}$.

In general, the design of a PID controller of linear system is broadly studied [21]. Nevertheless, the design of a PID controller of non-linear systems is not a trivial task. Tuning of a PID controller by using bifurcation theory is used for non-linear system [22]. From the feedback control strategy proposed in the closed loop system, the choice of the controller gains is realized so that ensures the desired convergence. The closed loop stability of the proposed strategy is stated by considering the convergence of the tracking errors.

The experimental tuning procedure is done by keeping in mind that the higher the proportional gain the lower the

TABLE I

PARAMETERS OF THE FBM-SSDF AND THE DC MOTOR

\begin{tabular}{ccc}
\hline \multicolumn{3}{c}{ FBM-SDF's parameters } \\
\hline$L_{1}=0.5593[\mathrm{~m}]$ & $J_{2}=0.00071\left[\mathrm{~kg} \mathrm{~m}^{2}\right]$ & $m_{2}=1.362[\mathrm{~kg}]$ \\
$L_{2}=0.102[\mathrm{~m}]$ & $J_{3}=0.0173\left[\mathrm{~kg} \mathrm{~m}^{2}\right]$ & $m_{3}=1.362[\mathrm{~kg}]$ \\
$L_{3}=0.610[\mathrm{~m}]$ & $J_{4}=0.00509\left[\mathrm{~kg} \mathrm{~m}^{2}\right]$ & $m_{4}=0.2041[\mathrm{~kg}]$ \\
$L_{4}=0.406[\mathrm{~m}]$ & $\phi_{2}=\phi_{3}=\phi_{4}=0[\mathrm{rad}]$ & \\
$r_{2}=0[\mathrm{~m}]$ & $r_{3}=0.305[\mathrm{~m}]$ & $r_{4}=0.203[\mathrm{~m}]$ \\
\hline \multicolumn{3}{c}{ Motor's parameters } \\
\hline$R=0.4[\Omega]$ & $L=0.05[\mathrm{H}]$ & $K_{f}=0.678[\mathrm{Nm} / \mathrm{A}]$ \\
$K_{b}=0.678[\mathrm{Vs}]$ & $B=0.226[\mathrm{Nms}]$ & $J=0.056\left[\mathrm{kgm} \mathrm{m}^{2}\right]$ \\
\hline
\end{tabular}

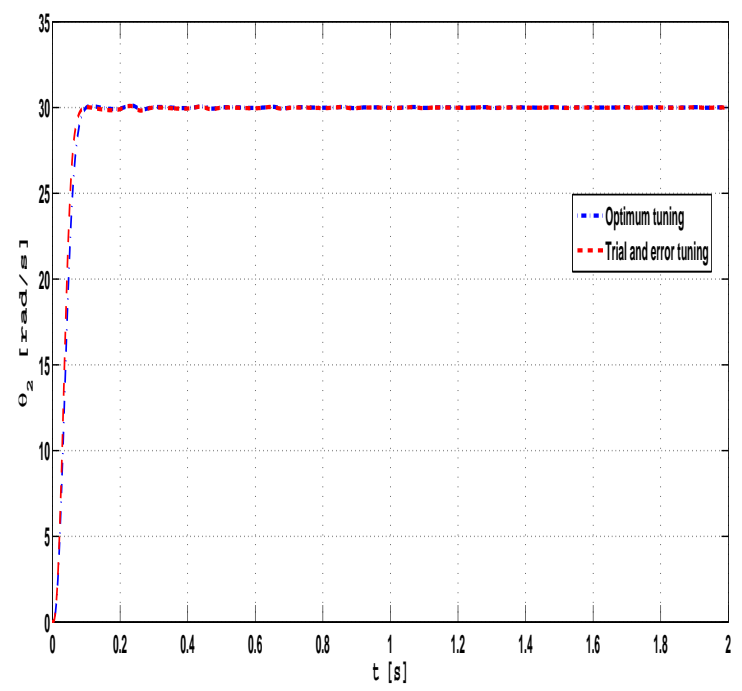

Fig. 5. Angular velocity of the crank with both tuning approaches: the optimum and experimental tuning

speed fluctuation and the steady-state error. On the other hand, excessively high proportional gains may lead to a large amount of overshoot if the derivative gain is not large enough. Additionally, increasing the derivative gain will decrease the overshoot, but the system response will be slower during the start-up period. The found gains need to fulfill the estimated performance, overshoot $\leq 1.5 \%$, steady-error $\leq 1.0 \%$ and rise time $\leq 0.1$ second. The resulting design variable vector with the experimental tuning is $\vec{p}_{e t}^{*}=[45.55,5.25,1]^{T}$ with a performance function value of $F\left(\vec{p}_{e t}^{*}\right)=0.2702$.

It is important to remark that in the experimental tuning procedure, several possible solutions were obtained,but they were not feasible from the optimization problem point of view. After several trials, we finally find the vector $\vec{p}_{e t}^{*}$ which fulfill the constraints in the optimization problem.

In Fig. 5, the angular velocity of the crank with both tuning approaches is shown. It is observed that in the optimum tunning, the angular velocity presents a deviation of $0.79 \%$ from the desired angular velocity. Also, the angular velocity deviation on the second case was $0.9 \%$. Finally, the rise time by each one of the approaches were $0.1 \mathrm{~s}$ and less of $0.1 \mathrm{~s}$, respectively. This indicates that the constraints in the dynamic optimization problem are satisfied.

On the other hand, the behavior of the control signal with the optimum design variable vector and with the experimental tuning is shown in Fig. 6. As it is observed, the control signal of the optimum approach has a lower overshoot than the second approach to reach the reference value of $30 \mathrm{rad} / \mathrm{s}$. This implies greater energy consumption by using the gains of the experimental tuning. Also, both approaches produce a control signal which compensates the nonlinear loads in order to reduce the angular velocity variation.

In Fig. 7, a zoom of the angular velocity of the crank 


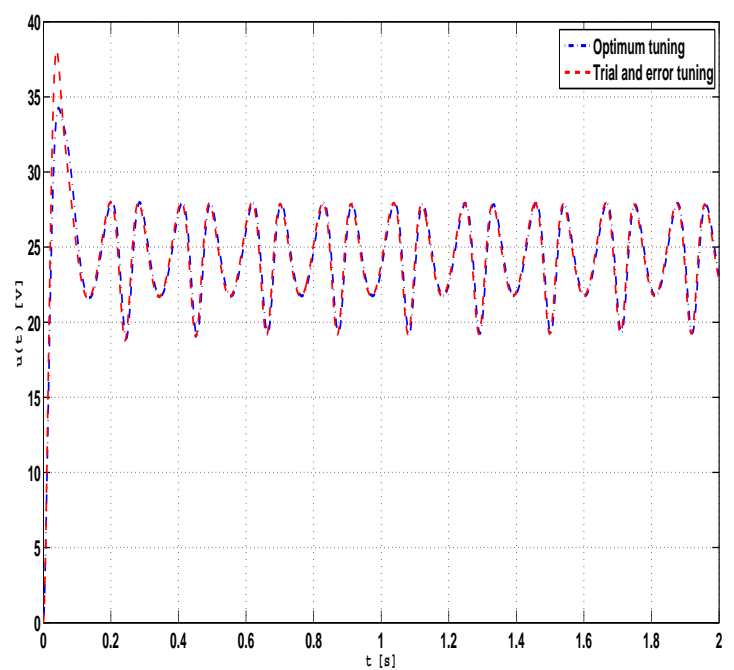

Fig. 6. Control signal dynamic behavior with both approaches

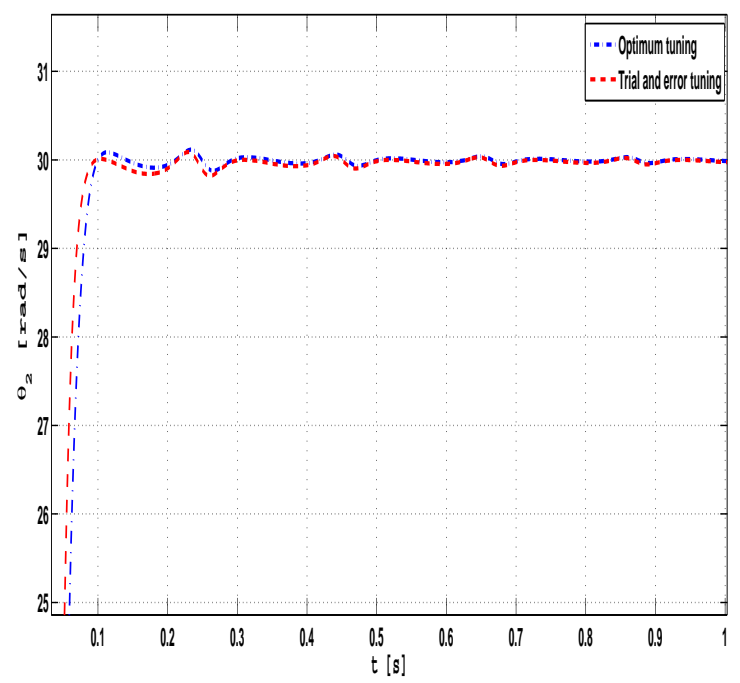

Fig. 7. Angular velocity of the crank between the time period of $0.05 \mathrm{~s}$ and $1 s$ with both approaches

with both approaches in the time period between $0.05 \mathrm{~s}$ and $1 s$ is shown. It is observed that in the experimental tuning procedure, the rise time of the angular velocity is less than the optimum approach. However, the steady state behavior of the optimum approach is most softly than the experimental tuning procedure.

It is important to comment the although both approaches produce good results, the best of them is the optimal one. In addition, the CHDE algorithm is successfully applied to tuning the PID controller without requiring a priori knowledge of the system and in the experimental tuning procedure is necessary this knowledge.

\section{CONCLUSION}

In this paper, the optimal gains of a PID controller for a four-bar mechanism with spring and damping forces is found by using a differential evolution algorithm with a constraint handling mechanism. In order to compare the performance of the system with the optimum control, an alternative experimental tuning procedure is carried out. The variation of the crank's velocity error for a four-bar mechanism with spring and damping forces is reduced by using both approaches. In addition, the rise time and the overshoot of the velocity signal are limited to be in an closed interval. However, simulation results of the closed-loop system show that the found optimal gains provide a better performance than the gains obtained by experimental tuning procedure.

Finally, the main advantage of using the differential evolution algorithm with a constraint handling mechanism for finding the optimum PID gains is that it does not require a priori knowledge of the system and it is easy to program it. Therefore the CHDE algorithm is becoming more used to solve this kind of nonlinear and discontinuous problems.

Further research involves the redesign of the structural and controller parameters considering the dynamic model and by using alternative evolutive algorithms.

\section{ACKNOWLEDGEMENTS}

The first author acknowledges support from CONACYT through a scholarship to pursue graduate studies at Instituto Politécnico Nacional. Authors acknowledge support from CONACyT through grant No. 182298 and support from COFAA and SIP of the Intituto Politécnico Nacional through grant No. 20131053 and 20131350.

\section{REFERENCES}

[1] R. Madangopal, Z. A. Khan, and S. K. Agrawal, "Biologically inspired design of small flapping wing air vehicles using four-bar mechanisms and quasi-steady aerodynamics," Journal of Mechanical Design, vol. 127 , pp. 809-815, 2005.

[2] M. E. Alfaro, D. I. Bolnick, and P. C. Wainwright, "Evolutionary dynamics of complex biomechanical systems: an example using the four-bar mechanism," Evolution, International Journal of Organic Evolution, vol. 58, no. 3, pp. 495-503, 2004.

[3] M. W. Westneat, "Feeding mechanics of teleost fishes (labridae; perciformes): A test of four-bar linkage models," Journal of Morphology, vol. 205 , no. 3, pp. 269-295, 1990.

[4] V. Parlakta, E. Söylemez, and E. Tanik, "On the synthesis of a geared four-bar mechanism," Mechanism and Machine Theory, vol. 45, no. 8, pp. 1142-1152, 2010.

[5] J. E. Shigley and J. J. J. Uicker, Theory of machines and mechanism. Mc. Graw Hill, 1995.

[6] J. Hrones and G. Nelson, Analysis of Four Bar Linkage. MIT Press and Wiley, 1951.

[7] M. Khorshidi, M. Soheilypour, M. Peyro, A. Atai, and M. S. Panahi, "Optimal design of four-bar mechanisms using a hybrid multi-objective ga with adaptive local search," Mechanism and Machine Theory, vol. 46, no. 10, pp. 1453-1465, 2011.

[8] N. Nariman-Zadeh, M. Felezi, A. Jamali, and M. Ganji, "Pareto optimal synthesis of four-bar mechanisms for path generation," Mechanism and Machine Theory, vol. 44, no. 1, pp. 180-191, 2009.

[9] R. A. Freeman and P. V. Kokotovic, Robust Nonlinear Control Design: State-Space and Lyapunov Techniques. Birkhäuser, 2008.

[10] J.-J. Slotine and W. Li, Applied Nonlinear Control. Prentice Hall, 1991. 
[11] J. Tao and J. P. Sadler, "Constant speed control of a motor driven mechanism system," Mechanism and Machine Theory, vol. 30, no. 5, pp. 737-748, 1995.

[12] D. T. Greenwood, Classical Dynamics. Dover Publications, INC, 1997.

[13] G. Tao and P. V. Kokotovic., Adaptive control of systems with actuator and sensor nonlinearities. John Wiley and Sons, 1996.

[14] J. Chiasson, Modeling and High Performance Control of Electric Machines. Wiley-IEEE Press, 2005.

[15] W. Hong, S. Wang, P. Li, G. Wozny, and L. T. Biegler, "A quasi-secuential approach to large-scale dynamic optimization problems," American Institute of Chemical Engineers, vol. 52, no. 1, pp. 255-268, 2005.

[16] M. G. Villarreal-Cervantes, C. A. Cruz-Villar, J. Alvarez-Gallegos, and E. A. Portilla-Flores, "Differential evolution techniques for the structurecontrol design of a five-bar parallel robot," Engineering Optimization, vol. 42, no. 6, pp. 535-565, 2010.

[17] E. A. Portilla-Flores, E. Mezura-Montes, J. Alvarez-Gallegos, C. A Coello-Coellod, C. A. Cruz-Villar, and M. G. Villarreal-Cervantes, "Parametric reconfiguration improvement in non-iterative concurrent mechatronic design using an evolutionary-based approach," Engineering
Applications of Artificial Intelligence, vol. 24, no. 5, pp. 757-771, 2011.

[18] M. G. Villarreal-Cervantes, C. A. Cruz-Villar, J. Alvarez Gallegos, and E. A. Portilla-Flores, "Kinematic dexterity maximization of an omnidirectional wheeled mobile robot: A comparison of metaheuristic and sqp algorithms," International Journal of Advanced Robotic Systems, vol. 9, no. 161, pp. 1-12, 2012.

[19] M. G. Villarreal-Cervantes, C. A. Cruz-Villar, J. Alvarez-Gallegos, and E. A. Portilla-Flores, "Robust structure-control design approach for mechatronic systems," IEEE/ASME Transactions on Mechatronics, 2013 On line version DOI: 10.1109/TMECH.2012.2208196.

[20] K. V. Price, R. M. Storn, and J. A. Lampinen, Differential evolution: A practical approach to global optimization. Springer, December 2005.

[21] I. Gyongy and D. Clarke, "On the automatic tuning and adaptation of pid controllers," Control Engineering Practice, vol. 14, no. 1, pp. 149-163, 2006.

[22] M. P. Polo, P. Albertos, and J. Ángel Berná Galiano, "Tuning of a pid controlled gyro by using the bifurcation theory," Systems \& Control Letters, vol. 57, no. 1, pp. 10-17, 2008 